\title{
Referees 2018
}

Published online: 14 February 2019

(C) Springer-Verlag GmbH Germany, part of Springer Nature 2019

We thank those who have given their time to referee for Diabetologia, all of whom are listed on our website (http://diabetologiajournal.org/about-the-journal/referees/). Special thanks are due to our key referees, listed below, who have each provided at least three timely and well-rated reports over the past year.

\begin{tabular}{|c|c|}
\hline Alman A; Tampa & Hunter CS; Birmingham \\
\hline Bansal A; Philadelphia & In’t Veld P; Brussels \\
\hline Bebu I; Rockville & Janssen JAMJL; Rotterdam \\
\hline Beck R; Tampa & Joseph JW; Kitchener \\
\hline Beeri M; New York & Kadowaki T; Tokyo \\
\hline Biden TJ; Sydney & Karpe F; Oxford \\
\hline Bouwens L; Brussels & Keymeulen B; Brussels \\
\hline Boyko E; Seattle & Lambers Heerspink HJ; Groningen \\
\hline Bryan N; Austin & Lau B; Baltimore \\
\hline Chatenoud L; Paris & Laybutt DR; Sydney \\
\hline Colhoun H; Edinburgh & Leslie RDG; London \\
\hline Cooper ME; Melbourne & Logue J; Glasgow \\
\hline Cusi K; Gainesville & Lowe W; Chicago \\
\hline Daniel C; Neuherberg & Ma RCW; Hong Kong \\
\hline de Galan BE; Nijmegen & Maeder MT; St Gallen \\
\hline Del Prato S; Pisa & Mäkinen V-P; Adelaide \\
\hline Dhatariya K; Norwich & Marx N; Aachen \\
\hline Eckel J; Düsseldorf & Marzban L; Vancouver \\
\hline Egan AM; Galway & McIntyre D; Queensland \\
\hline Eriksson O; Uppsala & Medina R; Belfast \\
\hline Evans M; Cambridge & Morris K; Cardiff \\
\hline Evans-Molina C; Indianapolis & Narendran P; Birmingham \\
\hline Fadini GP; Padua & Nauck MA; Bochum \\
\hline Freathy R; Exeter & Naziruddin B; Dallas \\
\hline Gerstein HC; Hamilton & Noordam R; Leiden \\
\hline Gillespie K; Bristol & Orchard TJ; Pittsburgh \\
\hline Gorus FK; Brussels & Palmer JP; Seattle \\
\hline Gregg E; Atlanta & Pasquali L; Barcelona \\
\hline Gunderson E; Oakland & Perng W; Ann Arbor \\
\hline Hamman RF; Denver & Pociot F; Herlev \\
\hline Harris RJ; London & Powers AC; Nashville \\
\hline Hill DJ; London & Retnakaran R; Toronto \\
\hline Hodson DJ; Birmingham & Rich SS; Charlottesville \\
\hline Hoffmann B; Düsseldorf & Roden M; Düsseldorf \\
\hline
\end{tabular}

Roep BO; Duarte

Rutter GA; London

Rutter MK; Manchester

Rutters F; Amsterdam

Saydah SH; Silver Spring

Scheen AJ; Liège

Sebastiani G; Siena

Sesti G; Catanzaro

Sharp S; Cambridge

Sigal RJ; Calgary

Simmons D; Campbelltown

Solini A; Pisa

Steculorum SM; Cologne

Taylor R; Newcastle upon Tyne

Thorens B; Lausanne

Tromp J; Groningen

Ussar S; Garching

Van Bergen T; Heverlee

van Dam RM; Singapore

van de Bunt M; Oxford

von Herrath MG; La Jolla

Welsh P; Glasgow

Wen L; New Haven

West DJ; Newcastle upon Tyne

White SL; London

Wilding JPH; Liverpool

Wilkinson-Berka JL; Melbourne

Williams AJK; Bristol

Wong FS; Cardiff

Woodward M; Sydney

Yu L; Aurora

Zhong V; Chicago

Publisher's note Springer Nature remains neutral with regard to jurisdictional claims in published maps and institutional affiliations. 